\title{
Effect of Stress on Spinodal Decomposition in Binary Alloys: Atomistic Modeling and Atom Probe Tomography
}

\author{
ALEXANDER DAHLSTRÖM, FREDERIC DANOIX, PETER HEDSTRÖM, \\ JOAKIM ODQVIST, and HELENA ZAPOLSKY
}

Self-organizing nanostructure evolution through spinodal decomposition is a critical phenomenon determining the properties of many materials. Here, we study the influence of stress on the morphology of the nanostructure in binary alloys using atomistic modeling and atom probe tomography. The atomistic modeling is based on the quasi-particle approach, and it is compared to quantitative three-dimensional (3-D) atom mapping results. It is found that the magnitude of the stress and the crystallographic direction of the applied stress directly affect the development of spinodal decomposition and the nanostructure morphology. The modulated nanostructure of the binary bcc alloy system is quantified by a characteristic wavelength, $\lambda$. From modeling the tensile stress effect on the A-35 at. pct B system, we find that $\lambda_{001}<\lambda_{111}<\lambda_{101}<\lambda_{112}$ and the same trend are observed in the experimental measurements on an Fe-35 at. pct $\mathrm{Cr}$ alloy. Furthermore, the effect of applied compressive and shear stress states differs from the effect of the applied tensile stress regarding morphological anisotropy.

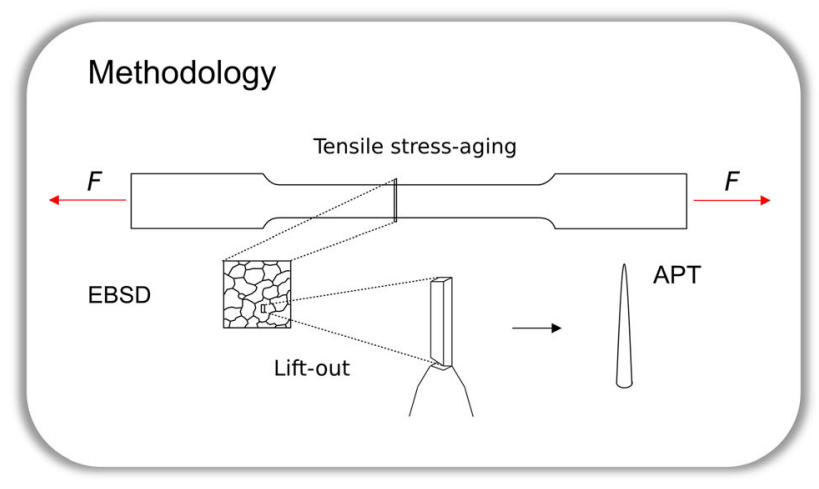

https://doi.org/10.1007/s11661-021-06467-3

(C) The Author(s) 2021

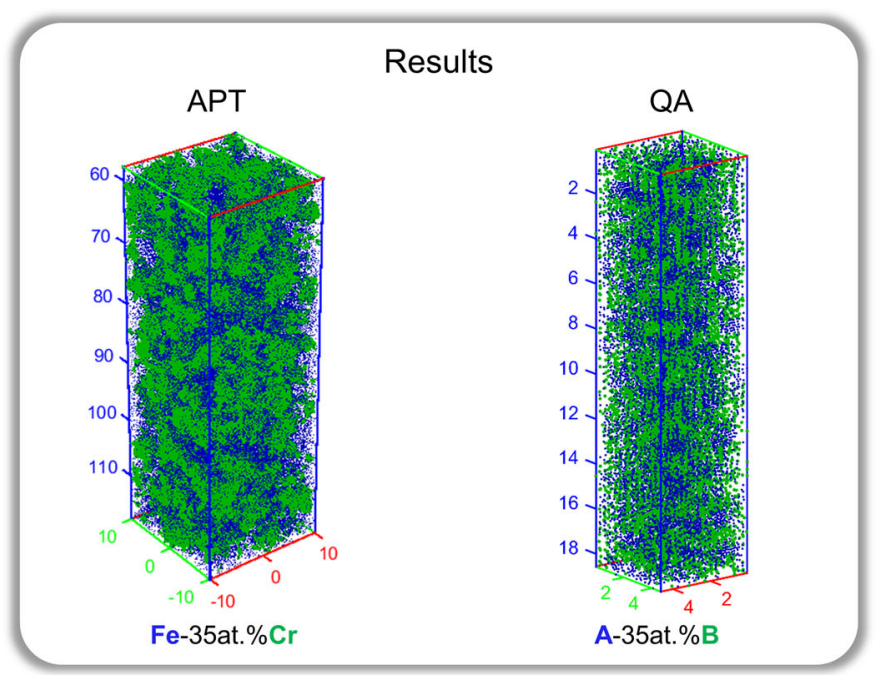

ALEXANDER DAHLSTRÖM is with the Normandie Université, Université de Rouen, INSA Rouen, CNRS, Groupe de Physique des Matériaux, 76000 Rouen, France, and the Department of Materials Science and Engineering, KTH Royal Institute of Technology, 10044 Stockholm, Sweden. Contact email: adahlstr@kth.se FREDERIC DANOIX and HELENA ZAPOLSKY are with the Normandie Université, Université de Rouen, INSA Rouen, CNRS, Groupe de Physique des Matériaux. PETER HEDSTRÖM and JOAKIM ODQVIST are with the Department of Materials Science and Engineering, KTH Royal Institute of Technology.

Manuscript submitted June 8, 2021; accepted September 20, 2021.

Article published online October 29, 2021
THE role of computational materials design is consistently increasing for the development of novel materials. The design process frequently adopts a hierarchic engineering approach since properties of materials at the macroscale are related to material structures on both the micro- and nanoscale. The bridging of the hierarchic scales is still challenging, for example, connecting first principles calculations to the arrangement of atoms at the nanoscale. However, there are theories for structural evolution that have been 
proven valid at the nanoscale. One such technique is phase-field crystal (PFC) modeling, which provides a mean to bridge the mentioned gap. ${ }^{[1,2]}$ A more general formulation of the PFC approach is the atomic density function (ADF) theory, which has been constructed by Khachaturyan. ${ }^{[3]}$ The continuum approach of the ADF theory adopts a pseudo-particle approach where atoms are built up by atomic fragments called fratons. This method was developed by Lavrskyi et al. ${ }^{[4]}$ to be able to model complex patterning and treat different types of phase transformations including diffusionless transformations, which involve short-range displacements of atoms.

Spinodal decomposition is a phase separation phenomenon found, for example, in $\mathrm{Ag}-\mathrm{Cu}$ nanoparticles, Ti-Al-N thin films, Fe-Mn, and Fe-Cr steels. ${ }^{[5-9]}$ This phenomenon is responsible for the self-organization of a nanostructure isostructurally. The phenomenon is beneficial in cutting tools with Ti-Al-N coatings, where the increased hardness gives an increased lifetime of the cutting tools, ${ }^{[10]}$ while, for example, in $\mathrm{Fe}-\mathrm{Cr}$ alloys, spinodal decomposition restricts dislocation motion and causes severe embrittlement of the alloys. ${ }^{[11,12]}$ The effect of stress is an important consideration in spinodal decomposition. The Ti-Al-N coatings produced by physical vapor deposition have large residual stresses and are also exposed to large stresses during cutting at high temperatures where decomposition occurs. In the case of stainless steel weldments, it is known that the existing residual stresses can enhance the decomposition kinetics. $^{[13]}$

The kinetics and morphology of spinodal decomposition, when exposed to applied tension, have been studied by Cahn. ${ }^{[14]}$ Although Cahn did not perform any simulations, he could deduce from his theoretical treatment that uniaxial tension or compression should result in a directionality of the decomposed morphology that was normal to the axis of the applied tension or parallel to the axis of the applied compression. In a more recent study by Thompson and Voorhees, ${ }^{[15]}$ elastic energy was assumed to be a function of the expansion difference between two coexisting phases constituting different solute concentrations. Their work considered stress in an inhomogeneous system as a function of composition-dependent elastic constants and an inhomogeneous strain field. Principal stress states were investigated to elucidate the relationship between the applied tension and the wavevector of the composition modulations. It was shown that the uniaxial loading both yields directional effects on the developing structure and can alter the limit of instability; that is, the temperature at which the decomposition process starts can be either suppressed or elevated dependent on the elastic properties of the system. The directional effect exhibited alignments of the nanostructure morphology perpendicular or parallel to the applied traction; this effect was found to be coupled to the sign of the applied strain, the solute expansion, and the difference between $\mathrm{C}_{11}-\mathrm{C}_{12}$ elastic constants.

Despite the many significant results obtained in the phase-field (PF) modeling of the spinodal decomposition in the $\mathrm{Fe}-\mathrm{Cr}$ system under external load, the understanding of the influence of atomic rearrangement at the atomic scale in the $\mathrm{Fe}-\mathrm{Cr}$ alloy under applied stress in the zone of spinodal decomposition is still an open question. In this work, we aim to elucidate the effect of the anisotropic elastic properties of the crystal structure in response to the applied stress through three-dimensional (3-D) simulations and direct experimental comparison. The binary system $\mathrm{Fe}-\mathrm{Cr}$ is a favorable model system to study the effect of stress on spinodal decomposition due to the small lattice mismatch between $\mathrm{Fe}$ and $\mathrm{Cr}$ and the relatively slow decomposition kinetics. In addition, $\mathrm{Fe}-\mathrm{Cr}$ is a technically important alloy used, for example, in reactor pressure vessels in the nuclear industry, ${ }^{[16,17]}$ where thermal embrittlement is of major concern. ${ }^{[18]}$ Furthermore, $\mathrm{Fe}-\mathrm{Cr}$ alloys are also important structural materials, for example, in the aviation industry, where complex load states may influence the spinodal decomposition and the related fatigue performance. ${ }^{[19]}$ Thus, understanding spinodal decomposition at an atomic scale beyond traditional thermodynamics is important. ${ }^{[20]}$ We apply an extended version of the continuum ADF theory, called the quasiparticle approach (QA), to model the spinodal decomposition of an elastically strained binary alloy at the atomic scale. ${ }^{[4]}$ The benefit of using QA is that we operate at a time and length scale that bridges the gap between DFT and MD simulations to mesoscopic techniques such as PF. This new approach opens a way to answer numerous outstanding questions concerning the atomistic mechanisms of the displacive phase transformation, ${ }^{[21]}$ formation of defects, dislocations, grain boundaries, ${ }^{[22,23]}$ vacancies, ${ }^{[24]}$ and crystallization, the formation of polymers due to the aggregation of monomers in their solution, ${ }^{[25]}$ pattern formation, ${ }^{[26]}$ etc. This list can be significantly extended. In other words, the QA allows description of the physical phenomena at the atomistic scale, keeping the diffusion time scale. Another benefit of the QA is that we do not have to introduce a complex expression for the elastic energy. As in the MD calculations, the interaction potential contains all of the information about the thermodynamic properties of the system and elastic interaction is integrated self-consistently in the model. The result is that we do not assume anything about the phase transformation itself, such as developing composition modulations and material-dependent interaction constants, which have major effects on the developing structure. Instead, we use a simple thermodynamic model that can capture the major effects found in the experiment.

This article is organized as follows. First, an overview of the QA with application to the binary system is presented, with special emphasis on the choice of model parameters. The QA is then applied to the model spinodal decomposition in the binary system under external stress. The effect of the tensile load along the [101], [111], and [112] crystallographic directions is examined. Finally, the simulation results are compared to the 3-D atom probe results. As will be shown in this article, the simulation results are in good agreement with the experimental observations. 
To describe the spinodal decomposition dynamics in binary alloys, we use the recently proposed quasi-particle approach (QA) ${ }^{[4]}$ In this approach, the size of the simulation grid is smaller than the distance between neighboring atoms, and we assume that each atom is a sphere comprised of a number of finite elements called fratons. The atomic displacement can be associated with the creation and annihilation of fratons at site $r$. The choice of model Hamiltonian should describe the interaction of fratons that results in both their "condensation" into atomic spheres and the redistribution of these spheres into the desired equilibrium atomic configuration. Then the main variable describing the atomic configurations in the QA is the fraton density probability function, $n_{\alpha}(r, t)$, which is the occupation probability of finding a fraton of the kind $\alpha(\alpha=1,2, \ldots, m)$ at the site $r$, where $m$ is the number of components in the system. In other words, $n_{\alpha}(r, t)$ is the probability that a given simulation grid point belongs to the atomic sphere of the kind $\alpha$. The temporal evolution of the density function of the fratons $n_{\alpha}(r, t)$ is described by the microscopic diffusion equation: ${ }^{[3]}$

$$
\frac{d n_{\alpha}(-r, t)}{d t}=\sum_{-r^{\prime}} \sum_{\beta=1}^{\beta=m} L_{\alpha \beta}\left(\vec{r}-\vec{r}^{\prime}\right) \frac{\delta F}{\delta n_{\beta}\left(\vec{r}^{\prime}, t\right)}
$$

Here, the summation is carried out over all points, $r^{\prime}$, of the computational grid approximating the continuum space. $L_{\alpha \beta}\left(r-r^{\prime}\right)$ is the matrix of the kinetic coefficients (i.e., exchange probabilities per unit time which in this work are: $L_{\mathrm{AA}}=L_{\mathrm{BB}}=1$ and $L_{\mathrm{AB}}=L_{\mathrm{BA}}=-0.5$ ), and $F$ is the free-energy functional. To guarantee the conservation of the number of atoms during the temporal evolution of the system, the condition $\sum_{-r} L_{\alpha \beta}(\vec{r})=0$ should be satisfied. The free energy

function can be written in the following form:

$$
\begin{aligned}
& F=\frac{1}{2} \sum_{-r^{\prime}, r^{\prime}} \sum_{\alpha=1}^{\alpha=m} \sum_{\beta=1}^{\beta=m} V_{\alpha \beta}\left(\vec{r}-\vec{r}^{\prime}\right) n_{\alpha}(\vec{r}) n_{\beta}\left(\vec{r}^{\prime}\right) \\
& +k_{B} T \sum_{-r}\left[\sum_{\alpha=1}^{\alpha=m} n_{\alpha}(\vec{r}) \ln n_{\alpha}(\vec{r})+\left(1-\sum_{\alpha=1}^{\alpha=m} n_{\alpha}(\vec{r})\right) \ln \left(1-\sum_{\alpha=1}^{\alpha=m} n_{\alpha}(\vec{r})\right]\right.
\end{aligned}
$$

where the first term describes the internal energy with an approximation of pair interaction and the second term represents the entropy term. $V_{\alpha \beta}\left(r-r^{\prime}\right)$ is the potential of the interaction of a pair of fratons, $\alpha$ and $\beta$. In the QA, $V_{\alpha \beta}\left(r-r^{\prime}\right)$ is constructed as a sum of two terms corresponding to a short-range and a long-range part of the interaction potential. The short-range interaction potential accounts for the spontaneous formation of atoms and prevents the overlapping of atoms, and the long-range interaction assures the auto assembly of atomic spheres in the desired crystal symmetry. The Fourier transformation (FT) of the interaction potential is

$$
\tilde{V}_{\alpha \beta}(k)=\frac{1}{N_{0}} \sum_{\vec{r}} V_{\alpha \beta}(\vec{r}) e^{-i \vec{k} \vec{r}}
$$

where the summation is carried out over all sites of the computational grid, and the wave vector, $k$, is defined at all quasi-continuum points, $k$, in the first Brillouin zone of the computational grid, that is, at all points $\left(N_{0}\right)$ in the $k$ space permitted by the periodic boundary conditions.

In accordance with Lavrskyi et al. ${ }^{[4]}$ the short-range interaction potential was chosen as the step function

$$
V^{\mathrm{Sr}}(r)=\left\{\begin{array}{clc}
-1 & \text { if } & R<r_{i} \\
\zeta & \text { if } & R \leq r_{i}+\Delta r \\
0 & \text { if } & R>r_{i}+\Delta r
\end{array}\right.
$$

where -1 accounts for the strength of the $i-i$ fraton agglomeration into atomic spheres, the constant $R$ is associated with the atomic radius (i.e., at $r<R$, fratons agglomerate due to the attractive forces), $\Delta r$ is the width of the contact repulsion part of the short-range interaction between atoms, and $\zeta$ is the height of the repulsive barrier ensuring structural integrity and repulsion between atoms.

For the long-range interaction, an isotropic potential is considered in this work by using a Gaussian distribution function. The number of wells and their position determines the crystal symmetry of the system. In this work, the binary bcc system is considered; it was previously shown that only one minimum potential is needed to reproduce this crystal symmetry. ${ }^{[4]}$ Then the isotropic long-range potential for the bcc crystal can be written as

$$
V_{\mathrm{LR}}(\vec{k})=-\exp \left(\frac{\left(k-k_{0}\right)^{2}}{2 \sigma}\right)
$$

The minimum of the Gaussian function is situated at $k_{0}=\frac{2 \pi}{a_{\mathrm{bcc}}} \sqrt{2}$, where $a_{\mathrm{bcc}}$ is the lattice parameter of the bcc crystal.

The elastic properties are essentially defined by the width of the Gaussian $\sigma$. Thus, the total interaction potential $\left.\mathrm{V}_{(} \alpha \beta\right)$ normalized by the energy density change determines the thermodynamic and mechanical properties of the system, which, in the binary case with $\alpha$ and $\beta$ fratons, is

$$
V_{\alpha \beta}(k)=\lambda^{\mathrm{Sr}} \frac{V_{\alpha}^{\mathrm{Sr}}(k)}{V_{\max }^{\mathrm{Sr}}-V_{\min }^{\mathrm{Sr}}}+\lambda^{\mathrm{Lr}} \frac{V_{\alpha \beta}^{\mathrm{Lr}}(k)}{V_{\max }^{\mathrm{Lr}}-V_{\min }^{\mathrm{Lr}}}
$$

where $\lambda^{\mathrm{Sr}}$ and $\lambda^{L r}$ are weight constants for short- and long-range interaction potentials, respectively. In these simulations, these constants were set to $\lambda^{\mathrm{Sr}}=1$ and $\lambda^{L r}=0.5 .^{[23,27]}$

To assure appropriate selection of the interaction potential parameters, for any given system, the elastic constant $C_{m n}$ can be calculated by $C_{i j k l}=\partial^{2} F / \partial \varepsilon_{i j} \partial \varepsilon_{k l}$. 
Strain, $\varepsilon$, is introduced by an infinitesimal displacement of the atoms through a deformation matrix, $\widehat{D_{k}}=\widehat{I}+\vec{\varepsilon}$, where $\widehat{I}$ is the identity matrix and $\vec{\varepsilon}$ is the strain vector directly defined in real space. Thus, the free energy of a deformed crystal can be evaluated by the change in the interaction potential from $V(r)$ to $V((\widehat{I}+\vec{\varepsilon}) r) \cdot{ }^{[27]}$ Then the elastic constants are calculated through the change in free energy of the system $\Delta F(\varepsilon)$ due to the infinitesimal deformation of the crystal.

For a cubic crystal, $C_{i j k l}$ is reduced to only three independent values: $C_{11}, C_{12}$, and $C_{44}$. To calculate these constants, three characteristic deformations are defined in compression/expansion: ${ }^{[2]}$ hydrostatic deformation $((x, y, z) \rightarrow(1-\varepsilon) x,(1-\varepsilon) y,(1-\varepsilon) z)$ for $C_{44}$, orthorhombic deformation $((x, y, z) \rightarrow(1+\varepsilon) x$, $(1-\varepsilon) y, z)$ for $C_{12}$, and monoclinic deformation $(((x, y, z) \rightarrow x+\varepsilon y, y, z))$ for $C_{11}$. Thus, the free energy associated with the corresponding deformations is given by

$$
\begin{array}{r}
F_{\text {cub }}(\varepsilon)=F+V_{0} \frac{3}{2}\left(C_{11}+2 C_{12}\right) \varepsilon^{2} \\
F_{\text {ortho }}(\varepsilon)=F+V_{0}\left(C_{11}-C_{12}\right) \varepsilon^{2} \\
F_{\text {monoc }}(\varepsilon)=F+V_{0} \frac{C_{44}}{2} \varepsilon^{2}
\end{array}
$$

where $F$ is the free energy of the unstrained system given by $F$ in Eq. [1]. Usually, to quantify the relation between the elastic constants of a cubic crystal, the Zener anisotropy ratio $\left(A_{\mathrm{Zn}}=C_{44} /\left(C_{11}-C_{12}\right)\right)$ is used. $A_{\mathrm{Zn}}=1$ corresponds to a fully isotropic material. It will be shown that the Gauss interaction potential used in our model can be used to reproduce the anisotropic properties of a bcc crystal. To calculate the elastic constants for the bcc $\alpha-\mathrm{Fe}$ crystal, the following set of parameters have been used: $\sigma=0.3, \mathrm{k}_{0}=-0.005, \mathrm{r}_{\mathrm{i}}=$ $6.4, \Delta r=6.4$, and $\zeta=4$. The computations are carried out for a $128 \Delta x^{3}$ computational cell with the lattice parameter $\mathrm{a}_{0}=16 \Delta \mathrm{x}$ and the fraton density $\rho_{\mathrm{A}}=0.125$. First, to estimate the free energy of the unconstrained bcc state $F_{0}$, small periodic fluctuations were introduced in the simulation domain and then the system was relaxed to an equilibrium bcc state using the microscopic diffusion Eq. [1]. To evaluate the elastic constants, hydrostatic, orthorhombic, and monoclinic deformations were applied to the equilibrium bcc state by changing the mesh spacing, as mentioned previously. Then the free energy curves as a function of strain were

Table I. Elastic Constants of Bcc Fe From ADF Modeling and Ab Initio (GPa)

\begin{tabular}{llllll}
\hline & \multicolumn{1}{c}{ Type } & $C_{44}$ & $C^{\prime}$ & $B$ & $A_{Z n}$ \\
\hline This work, Gauss potential & ADF & 116 & 49 & 130 & 2.37 \\
Müller et al..$^{[28]}$ theory & ab initio & 96 & 65 & 190 & 1.48 \\
Müller et al..$^{[28]}$ experiment & - & 116 & 43 & 169 & 2.69 \\
Mendelev et al. ${ }^{[29]}$ & ab initio & 116 & 49 & 178 & 2.37 \\
\hline
\end{tabular}

calculated and the values of elastic constants were estimated using the second derivative of the free energy. The obtained results are presented in Table I; included in the table are also ab-initio and experimental data ${ }^{[18,19]}$ for comparison.

The work of Müller et al. ${ }^{[28]}$ is based on DFT calculations; however, in the article by Mendelev et al.,$^{[29]}$ the embedded-atom method potential was used. Hence, there are differences between the predicted and the experimentally measured values of the elastic constants in Table I. Using the ADF approach, proper elastic constants of bcc Fe can be obtained through an atomic interaction defined by either a Gauss potential (as in this work) or by fitting to a structure factor determined by X-ray diffraction, as in Kapikranian et al. ${ }^{[2]}$ The reproduction of $\mathrm{Cr}$ elastic constants is cumbersome as $\mathrm{Cr}$ elastic is a brittle material with negative Cauchy pressure that requires a negative curvature of the embedding function. Hence, Pasianot et al. ${ }^{[30]}$ only managed to reproduce $\mathrm{Cr}$ by a polynomial pair potential, something which was not done in this work due to the complexity of the potential. The anisotropy is of high importance as the influence of crystallographic orientation is the main objective in this work. Thus, it is more important to obtain robust values for $\mathrm{Fe} C_{44}$ and $C$, which will dictate the elastic properties of this system.

For infinitesimal displacements where the material exhibits linear elastic behavior, Hooke's law $(\widehat{\sigma}=\widehat{C} \widehat{\varepsilon})$ is generally assumed valid. In this work, the stress tensor $\widehat{\sigma}$ and strain tensor $\widehat{\varepsilon}$ are both field tensors of rank 2 coupled by a material tensor $\widehat{C}$ of rank 4 characterizing the elastic compliance of the material. Then, it is possible to use the stiffness tensor $\widehat{S}$ to relate stress, stiffness, and strain $(\widehat{\varepsilon}=\widehat{S} \widehat{\sigma})$. The position of an atom, initially situated at point $r$, can after deformation be defined using the three-by-three deformation matrix $\widehat{D}$. The atom position vector after deformation is given by $\widehat{r}^{\prime}=\widehat{D} \widehat{r}$. Similarly, in Fourier space, the relation between the vectors $k$ and $k$, corresponding to the undeformed and deformed structures, can be written using the following expression:

$$
\vec{k} \vec{r}^{T}=\vec{k}\left(\hat{D}^{-1} \hat{D}\right) \vec{r}^{T}=\left(\vec{k} \hat{D}^{-1}\right)\left(\hat{D} \vec{r}^{T}\right)=\vec{k}^{\prime} \vec{r}^{T}
$$

In the simulations, $\vec{k} \vec{r}^{T}=\vec{k}^{\prime} \vec{r}^{T}=2 \pi$, where $\vec{k}$ is a row vector and $\vec{k}^{T}$ is a colon vector. From this expression, we can relate the displacement of the wave vector in reciprocal space to the deformation matrix in real space by $\vec{k}^{\prime}=\vec{k} \widehat{D}^{-1}$. Thus, the deformation matrix becomes

$$
\hat{D}^{-1}=\left(\begin{array}{ccc}
1 & 0 & 0 \\
0 & 1 & 0 \\
0 & 0 & 1 /(1+\varepsilon)
\end{array}\right)
$$


In this case, uniaxial deformation in the [001] direction is considered; however, any $3 \times 3$ deformation matrix can be introduced, and through the introduction of a 3-D rotation matrix, any crystal can be simulated, rotated, and deformed. In this work, the rotation matrix is of elasticity is here defined by the point after which $\Delta F$ starts to oscillate by higher-order terms. It is known that the anisotropic properties of the crystal correlate with anisotropic plastic deformation. ${ }^{[31]}$ Thus, the $\Delta F$ ratio as a function of the uniaxial tensile and shear deformations is obtained by $\Delta F\left(\varepsilon_{\mathrm{k}}\right)=D_{\text {mono. }}\left(\varepsilon_{\mathrm{k}}\right) / D_{\text {otho. }}\left(\varepsilon_{\mathrm{k}}\right)$, where $\varepsilon_{k}$

$$
\begin{aligned}
\Re(\varphi, \theta, \gamma) & =R_{100}(\varphi) \times R_{010}(\theta) \times R_{001}(\gamma) \\
& =\left(\begin{array}{ccc}
\cos \gamma \cos \theta & -\sin \gamma \cos \varphi+\cos \gamma \sin \theta \sin \varphi & \sin \gamma \sin \varphi+\cos \gamma \sin \theta \cos \varphi \\
\sin \gamma \cos \theta & \cos \gamma \cos \varphi+\sin \gamma \sin \theta \sin \varphi & -\cos \gamma \sin \varphi+\sin \gamma \sin \theta \cos \varphi \\
-\sin \theta & \cos \theta \sin \varphi & \cos \theta \cos \varphi
\end{array}\right)
\end{aligned}
$$

Hence, the strain is introduced by an infinitesimal displacement of the atoms sitting on the lattice; the displacement is inherently dependent on the discretization of the simulation grid. In this work, the morphology is of importance; thus, a trade-off between computational cost and discretization is made. In this work, the compromise was $\mathrm{a}_{0}=8 \Delta \mathrm{x}$.

To relate the simulated and real deformation, the length ratio $L / L_{0}$ (current length $L$ over initial length $L_{0}$ ) is in this case described by a Green-Lagrange strain tensor, as it accounts for first- and second-order elastic energy contributions given by the mean square displacement. Thus, the displacement of atoms represents strain that leads to a deformation of the simulated volume. The Green-Lagrange description of the deformed simulation volume provides a better scaling to the experiments, and it more easily defines the minimum of the second-order equation, Eq. [7], that defines the elastic limit. The experimental macroscopic deformation and strain were obtained through tensile testing of the Fe-35 at. pct $\mathrm{Cr}$ alloy.

As previously stated for the definition of the elastic limit in our simulations, we assume that the linear elastic behavior with the relation $\Delta \mathrm{F} \propto \varepsilon^{2}$ holds. Thus, the limit

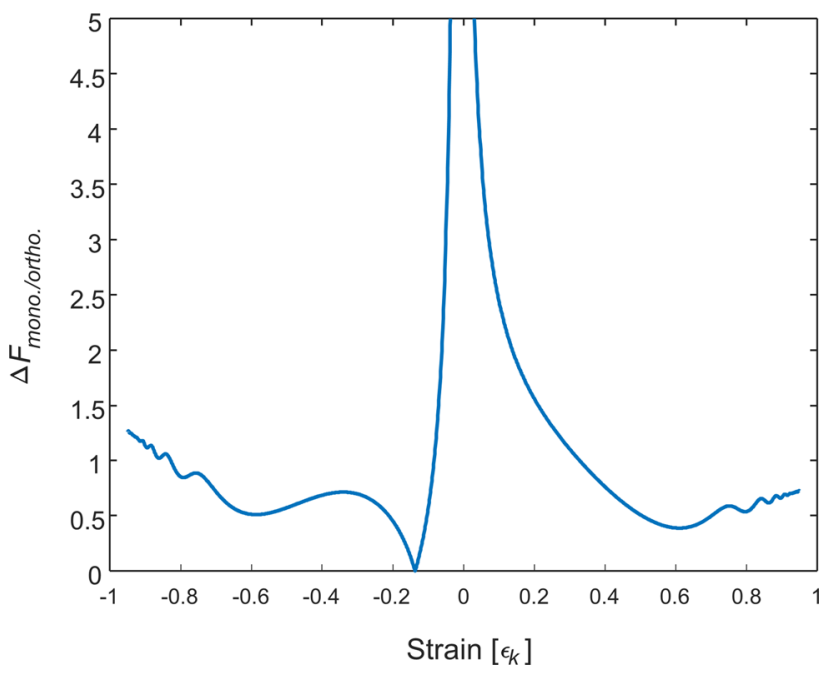

Fig. 1-Change in free energy because of strain, $\varepsilon_{k}$ (atomic displacement), in reciprocal space $\mathrm{k}$. is strain or, more accurately, displacement introduced in $k$ space; the result is shown in Figure 1. This stress state is related to the Zener anisotropy ratio and, therefore, related to the effective shear.

The deformation in our simulations is introduced in reciprocal space; thus, for $\varepsilon_{k}>0$, the system is compressed, and if $\varepsilon_{k}<0$, the system is elongated (Figure 1). For bcc $\mathrm{Fe}$, the elastic limit is easily defined in compression by the minimum in Figure 1 at $\varepsilon_{k}=0.61$ as it thereafter starts to oscillate. The equivalent volume dilatation in tension is given by $\varepsilon_{\mathrm{k}}=-0.213$. However, the minima and subsequent deviation from linear elastic behavior in tension begins just after $\varepsilon_{\mathrm{k}}=-0.195$, which corresponds to 27.4 pet volumetric deformation. This shows a difference in response upon tensile or compression loading of the system. Similar asymmetric stress behavior has been observed by Huang et al., ${ }^{[32]}$ where the difference was attributed to the change in Poisson's ratio. Thus, a discontinuous behavior may be recognized as a change in the crystallography due to deformation. Compression, therefore, is used to define the elastic limit and normalize the load scale as it is well defined and continuous.

The effect of stress on spinodal decomposition was also studied experimentally for a binary $\mathrm{Fe}-35$ at. pct $\mathrm{Cr}$ alloy. The model alloy was received in hot-rolled and air-cooled condition. Tensile specimens were produced according to ASTM E-8, and the tensile axis was aligned parallel to the transverse rolling direction. The samples were cut to 2-mm sample thickness using a diamond wire saw. The samples were aged at $525^{\circ} \mathrm{C}$ for 100 hours under an applied uniaxial tensile load of $\sigma=235 \mathrm{MPa}$ in an MTS Landmark tensile rig. The yield strength of the specimens was $\sigma_{0.2}=310 \mathrm{MPa}$ at room temperature. To prepare APT needles along certain crystallographic directions, the gage section of the tensile specimen was analyzed by electron backscatter diffraction after aging. Specific grain orientations were selected, and site-specific APT needles were prepared by FIB. At least three APT needles per orientation were analyzed per condition, and this forms the basis for the results presented here. Statistical analysis was performed on representative subvolumes for comparison. The APT analyses were performed on a CAMECA ${ }^{\circledR}$ LEAP 4000 HR instrument at $50 \mathrm{~K}$, pulse fraction $20 \mathrm{pct}$, repetition rate $200 \mathrm{kHz}$, 


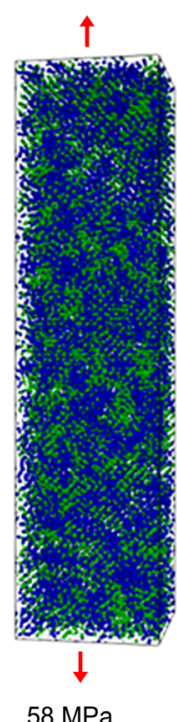

$58 \mathrm{MPa}$

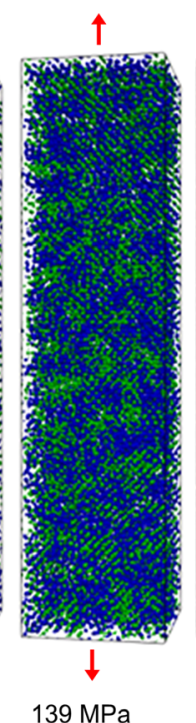

$139 \mathrm{MPa}$

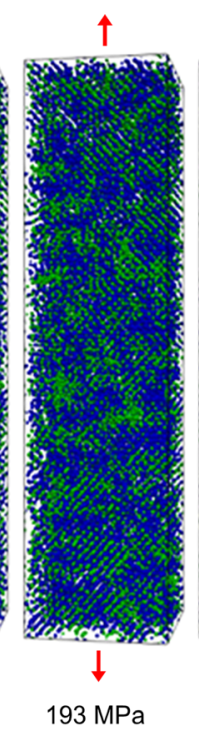

(a)

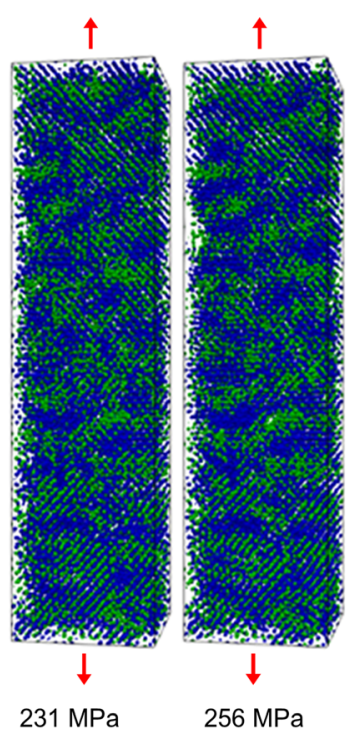

$256 \mathrm{MPa}$

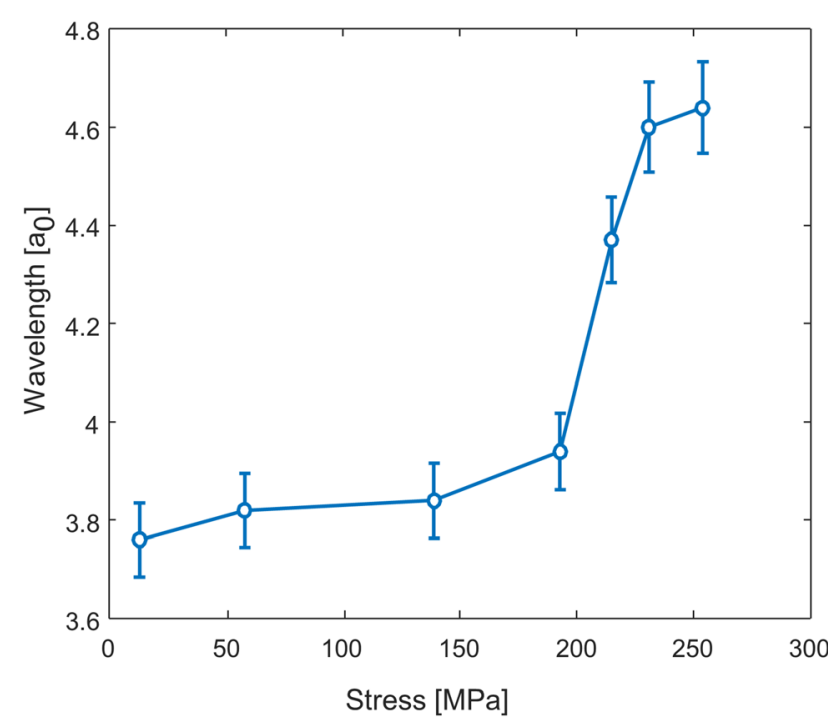

(b)

Fig. 2-(a) Microstructure evolution during the spinodal decomposition in the A-35 at. pct B system at $565{ }^{\circ} \mathrm{C}$. (b) Different external tensile loads applied along the [001] direction as indicated by the red arrows and the wavelength evolution as a function of stress in the direction of the applied load (Color figure online).

and evaporation rate 0.3 pct. The 3 -D reconstructions of the tips were performed using IVAS $₫ 3.8$ according to the reconstruction scheme by Moody et al. ${ }^{[33]}$

The influence of an applied load on Fe-Cr morphology was previously studied using various simulations and experiments. ${ }^{[7,13,34]}$ In this work, the 3-D experimental atomic-scale comparison is key; thus, we solely work with uniaxial tensile loads to simplify the experiments. The strength measured at room temperature, furthermore, is expected to be reduced at elevated temperatures. To assure that experiments are performed purely within the spinodal regime, experimental aging is made at $525^{\circ} \mathrm{C}$, where one can expect $\sim 23$ pct reduction in yield strength. ${ }^{[35]}$ The simulations are performed at a slightly higher temperature than the experiment; since the simulation kinetics is very sensitive to the selection of temperature, lowering the temperature would dramatically increase the simulation time. Thus, the loads of $235 \mathrm{MPa}$ in experiments and $231 \mathrm{MPa}$ (or $\left.\varepsilon_{\mathrm{k}}=-0.171\right)$ in simulations are used for the direct comparison. Furthermore, we assume that the Taylor model holds, meaning all grains experience the same level of strain in the polycrystalline alloy. ${ }^{[36]}$

Using the simulations, we start by applying different stress levels in the [001] direction using a pure tensile load. Then selecting the load equivalent to the one used in the experiment, the crystal is rotated in the simulation box so the different principal directions of the bcc crystal are aligned in the $\mathrm{z}$-axis of the simulation box. In addition, the response of the system to different stress states is simulated as well.

Figure 2 shows the microstructural evolution of the binary A-35 at. pct B system as a function of tensile load applied along the [001] direction. The minimum strain to trigger a measurable response on the system is in this case equivalent to $\sim 19$ pct of the elastic limit. The lack of elastic energy contribution at lower strain can be attributed to softening since these simulations are performed at elevated temperatures to favor the kinetics. The critical simulation temperature of instability $\left(T_{\text {critical }}\right)$ is defined in the simulations by the potential $k_{0}=0.00$; then the undercooling is dependent on the negative depth of the potential and the resulting simulation temperature is easily compared to the critical value. The undercooling in this work is $0.9912 \times T_{\text {critical }}$ or close to $565^{\circ} \mathrm{C}$ in experimental values. This information is used to calibrate the scale for the load level, that is, assuming we have a 19 pct reduction of the elastic limit for the A-35 at. pet B system at $565^{\circ} \mathrm{C}$ in comparison to room temperature. Thus, with an increased load to $\sim 98$ pet of the elastic limit of $256 \mathrm{MPa}$ at $565^{\circ} \mathrm{C}$, a pronounced alignment of the B-rich region appears. Given the system's response to the external load given in Figure 1, 90 pct of the defined elastic limit, or $\varepsilon_{\mathrm{k}}=-0.192$, is a more suitable choice for the maximum allowed stress in tension as the abrupt change in behavior may be attributed to a deformation of the crystal.

In addition, Figure 2 shows an alignment of the decomposed structure directly related to the level of the applied load. This alignment naturally affects the wavelength of the structure in the load direction. The morphological effect is expected as the perpendicular directions would contain lower elastic energy during high loads.

Based on the initial results, we expand our simulations to investigate the influence of uniaxial compression along the [001] direction (Figure 3(b)) and shear of the $\{011\}$ planes (Figure 3(c)) on the morphology; the applied strain was again fixed to $231 \mathrm{MPa}$ equivalent. The simulation results are shown in Figure 3(b), which produces a strong morphological anisotropy during compression. In the case of shear loading, it is possible to see some alignment at a 45 deg angle from the applied load.

The effects of tensile $231 \mathrm{MPa}$ load along the [101], [111], and [112] crystallographic directions are illustrated in Figures 3(d) through (f). Under the load 


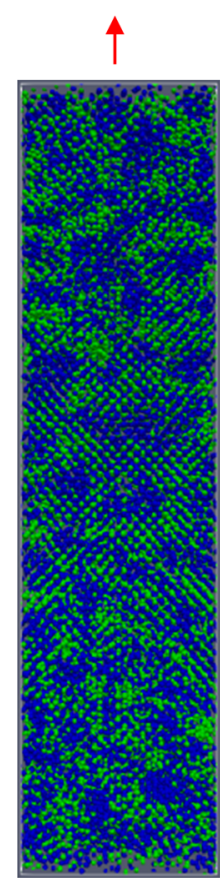

(a)

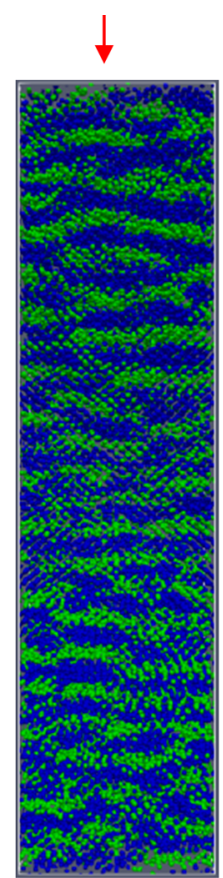

(b) $\uparrow$

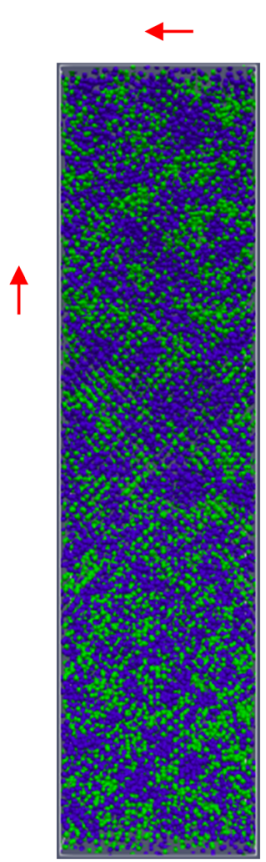

(c)

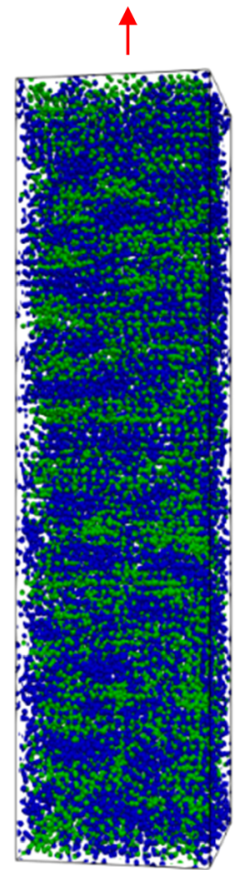

(d) $\downarrow$

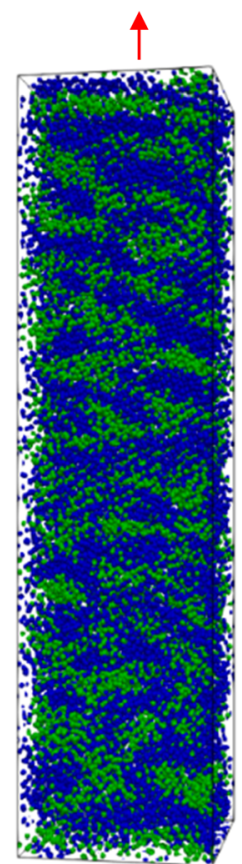

(e)

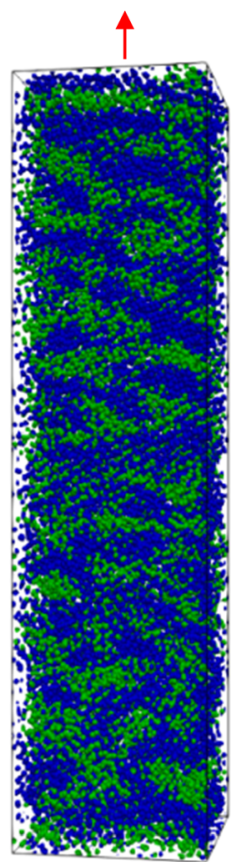

(f) $\downarrow$

Fig. 3-(a) through (c) Effect of $231 \mathrm{MPa}$ tensile, compressive, and shear stress on morphology. (d), (e), and (f) Effect of $231 \mathrm{MPa}$ tensile load as a function of crystallographic orientations [101], [111], and [112], respectively.

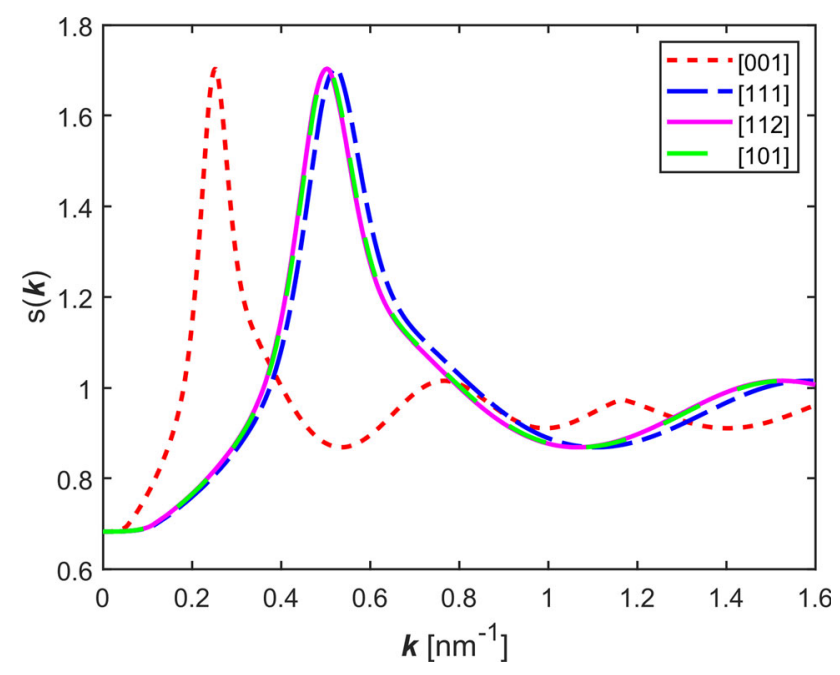

Fig. 4 - Simulated structure factor $s(k)$ for bcc crystals under an applied load.

applied to the [101] direction, a more pronounced alignment of the structure perpendicular to the load is observed. The $\{101\}$ plane is a close-packed plane; thus, a greater orientation effect in relation to the [001] direction can be expected. However, when the load is applied along the $\{111\}$ and $\{112\}$ directions, the alignment of the microstructure starts to rotate in relation to the applied load. To quantitatively characterize this effect, the structure factor $s(k)$ was calculated for the principal directions in their equilibrium state. The result is shown in Figure 4, where $s(k)$ is obtained through the FT of the density probability function $n(k, t)$, also used to describe the primitive unit cell. In Figure 4, we can clearly distinguish the effect of the rotated crystal symmetry and applied load on the peak position. Thus, the first peak of $s(k)$ determines the nearest neighboring atom in the direction of the static concentration wave that is the equilibrium solution to our simulation. As an example, for the 001 orientation, we apply $231 \mathrm{MPa}$ through $-0.192 \AA$ displacement of the atomic position in reciprocal space. In Figure 4, the first peak is located at $1.703 \AA(1.703 \AA \times 1.192=2.03 \AA$, i.e., interatomic plane distance of $\{011\}$ bcc Fe). Thus, applying load in the [001] orientation favors the decomposition in the [0-11] and [110] directions. Similarly, applying load in the [110] orientation favors decomposition in the [001] direction, and because [001] orientation is a soft direction, we see a more pronounced alignment of the structure. In the case of [111] and [112] orientations, we can see that their $\mathrm{s}(k)$ tends to align themselves in a similar way as the 101 orientation. This gives rise to an approximately $30 \mathrm{deg}$ rotation of the structure in real space, as seen in Figure 3.

In this section, simulating the response to the tensile load was mainly performed in the elastically soft [001] direction for load scale calibration and to potentially maximize the effect of the applied stress. ${ }^{[14,37]}$ This is a known effect on $\mathrm{Fe}-\mathrm{Cr}$ morphological anisotropy, previously modeled by phase-field methods, ${ }^{[7,38,39]}$ where morphological effects only were observed at very high load levels. The advantage of the QA approach compared to using the phase-field methods is the ability to consider externally applied strains where elastic properties are independent from the developing composition modulations. 

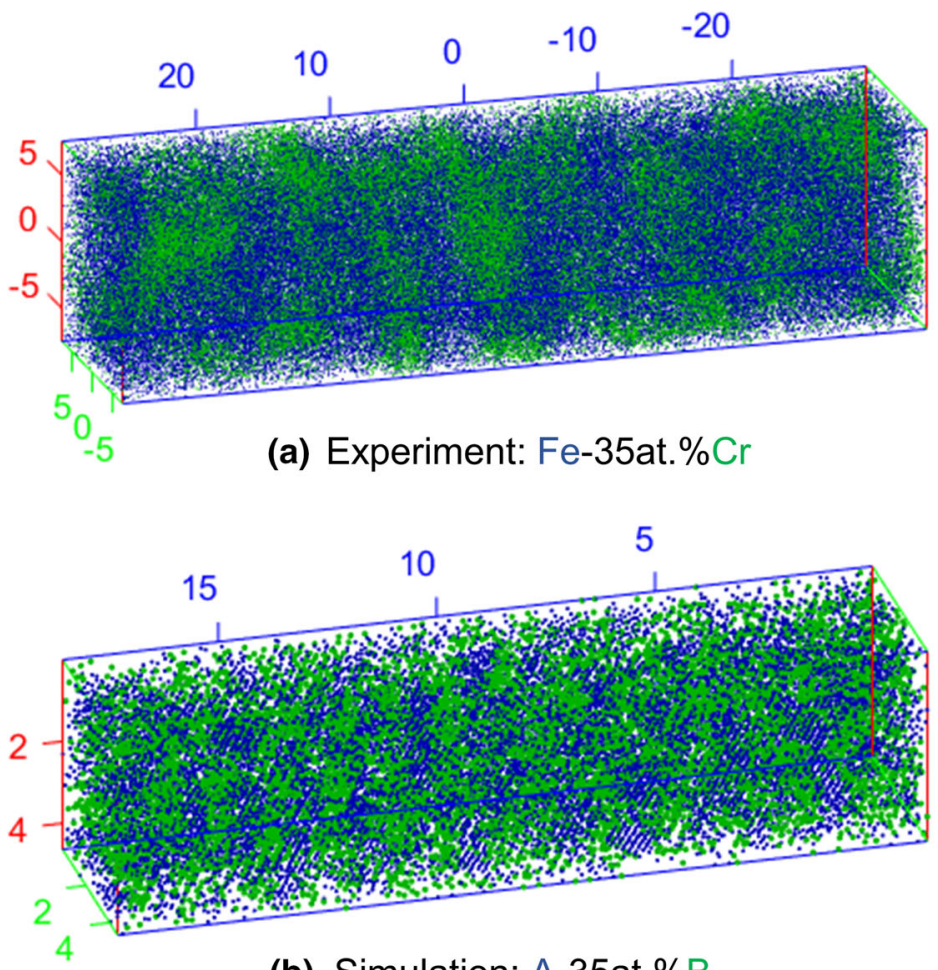

Simulated diffraction

(b) Simulation: A-35at.\%B

Fig. 5-Visual comparison between (a) APT sample aged under $235 \mathrm{MPa}$ tensile load in the [001] direction at $525^{\circ} \mathrm{C}$ and $(b)$ simulation results from QA $231 \mathrm{MPa}$ tensile load in the [001] direction at $565^{\circ} \mathrm{C}$. Units: $\mathrm{nm}$.

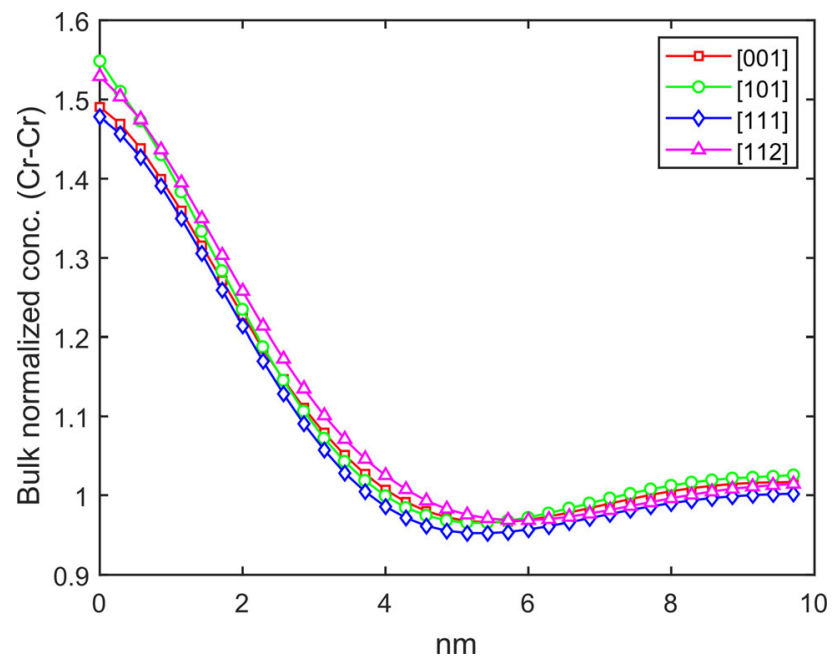

Fig. 6-Stress aging of Fe-35 at. pct Cr, 100 h, $235 \mathrm{MPa}$ tensile load analyzing different grain orientations by APT; analysis volume $20 \times$ $20 \times 60 \mathrm{~nm}^{3}$.

Focusing the analysis on the $235 \mathrm{MPa}$ tensile load presented in Figure 5, there is an apparent difference in the volume fraction of $\alpha^{\prime}$ represented by the green atoms for QA and APT. The volume fraction $\alpha^{\prime}$ obtained through the lever rule in QA is roughly $\sim 0.40$, while in APT, it is closer to $\sim 0.1$. This difference is due to the construction of the miscibility gap in the model and partially because we run the model to equilibrium, which is not reached in experiments. In APT, $\alpha^{\prime}$ near equilibrium composition consists of $\sim 80$ at. pet $\mathrm{Cr}$ and $\alpha$ contains $\sim 14$ at. pet $\mathrm{Cr},{ }^{[40]}$ while in QA, we model atoms that are considered pure elements with an associated lattice site occupation probability. It should also be noted that the reason we accept a higher volume fraction in QA is that the simulated volume is much smaller; if the volume fraction is much smaller, we are not be able to quantify the wavelength of the spinodal structure in real space. In Figure 6(b), the QA diffraction pattern is shown as the probability distribution intensity $I(k)=$ $I(k)=\tilde{n}(\mathrm{k}, \mathrm{t}) \cdot \tilde{n}(\mathrm{k}, \mathrm{t})^{*}$. In this figure, we can clearly distinguish the fundamental diffraction spots of the bcc lattice and diffuse scattering around some main spots. This pattern is used in comparison with the APT detector hit density to make sure the right crystallographic orientation is analyzed; calculated stereographic projections in the Appendix were used for APT reconstruction calibration.

In Figure 5(a), there is a tendency of $\alpha^{\prime}$ to elongate perpendicular to the applied stress seen experimentally; in that case, the load was applied in the [001] direction, which resulted in $\alpha^{\prime}$ alignment in the [010] direction. The experimental aging time was 100 hours; still, the nanostructural comparison of the results shows good agreement in the morphological alignment between APT and QA. The main morphological discrepancy is a higher degree of anisotropic morphological alignment in QA, presumably attributed to the difference in volume fraction $\alpha^{\prime}$ between the modeling and experiment. Figure 5 also shows the simulated diffraction pattern and detector hit density map of the [001] direction, in which the external load was applied. Naturally, in the experiment, there are a few degrees deviation from the 
true calculated projections, which might slightly influence the results. Still, without a perfect APT/QA comparison based on the morphology and behavior dependent on the applied load, we obtain good reproduction of the nanostructure between the experiment and modeling. Note in the comparison that the elastic properties of $\mathrm{A}$ and $\mathrm{B}$ elements in the A-35 at. pct B system are only fitted to $\alpha$-Fe for the main component A. Thus, $\alpha-\mathrm{Fe}$ is the base element of the simulated system and of the Fe-35 at. pct $\mathrm{Cr}$ alloy, which is used for comparison. Hence, no difference in elastic behavior between A and B atoms is modeled, even though that is not true for the real $\mathrm{Fe}-\mathrm{Cr}$ systems.

Figure 5 illustrates the visual correlation between our model and the experimental work; only tensile load is considered for this comparison. High $\alpha^{\prime}$ morphological anisotropy for very high tensile load levels has been seen before by APT in duplex Fe-Cr weld alloys, ${ }^{[13]}$ where the question regarding the thermal effect on the local stress state due to the difference in thermal expansion was unresolved. This issue is not of concern in this analysis as these alloys are fully ferritic binary $\mathrm{Fe}-\mathrm{Cr}$ alloys with conservative load levels. The conservative load level is presumably the reason no significant morphological anisotropy could be quantified in these experiments. However, there is good agreement in the system's response to the applied load regarding its effect on the characteristic wavelength. Experimental investigations in this case show accelerated fluctuations in $\mathrm{Cr}$ composition amplitudes dependent on crystallographic orientation, which can be seen in Figure 6.

The bulk normalized concentration at zero distance is evaluated by the radial distribution function (RDF), which, in this case, relates to the amplitude of $\mathrm{Cr}$

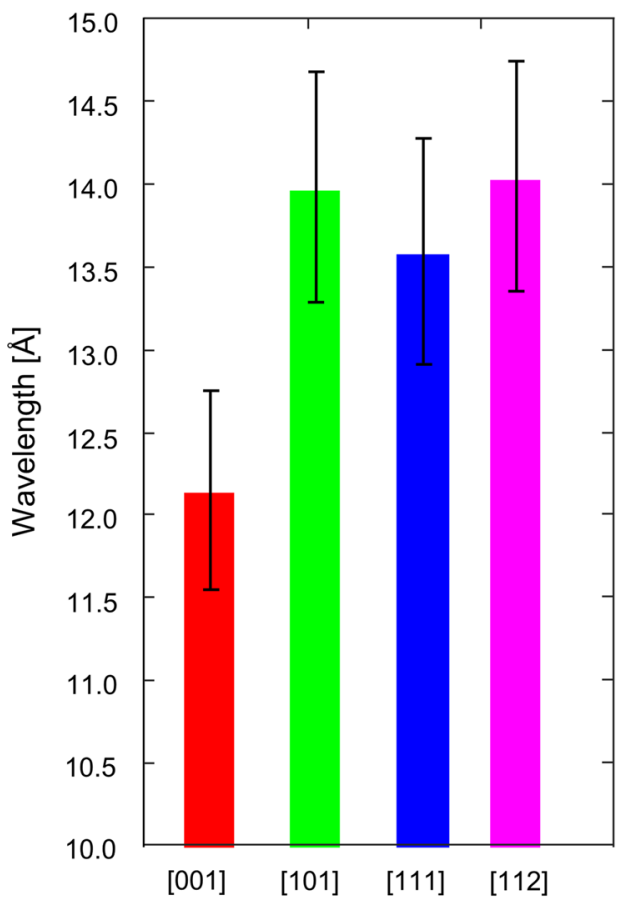

(a) fluctuations. ${ }^{[41]}$ In Figure 6, one can clearly evaluate $[111]<[001]<[112]<[101]$ even though the differences are small. The $\mathrm{Cr}$ amplitude is known to be related to the degradation of mechanical properties. ${ }^{[11]}$

The difference in morphology of the nanostructure after applying tensile load along the [001], [101], [111], and [112] directions is very small in both APT and QA. However, in the trend of the characteristic wavelength $\lambda$ obtained through autocorrelation in real space, we find from the modeling that $\lambda_{001}<\lambda_{111}<\lambda_{101}<\lambda_{112}$, and in the experiments, we obtain the same results from RDF: $\lambda_{001}<$ $\lambda_{111}<\lambda_{101}<\lambda_{112}$. These results are presented in Figure 7 .

In APT, the $\lambda_{001}$ and $\lambda_{111}$ are relatively similar, while the close-packed slip plane of [101] and the [112] slip plane both produce longer wavelengths $\lambda_{101}$ and $\lambda_{112}$, respectively. A similar trend is observed by QA. However, in QA, the supposedly soft orientation [001] produced a much shorter wavelength, even though we expect the opposite given $s(k)$ in Figure 4. Still the results in Figure 7(a) confirm the results shown in Figures 3 and 5. Thus, this result may be attributed to a visualization effect in $3-\mathrm{D}$ and the subsequent voxelization of real space to calculate the wavelength $\lambda$ by autocorrelation. By visual inspection of the QA volumes in Figures 3(b) and 2, one would expect the [001] wavelengths to be longer than the others. Thus, one should be careful to draw definitive conclusions given the relatively small difference even though the trend seems obvious.

In phase-field modeling of phase separation in anisotropic elastic bodies, anisotropic alignment of the $\alpha^{\prime}$ phase is derived from the Cahn-Hilliard theory. ${ }^{[38,39]}$ Zhou et al. ${ }^{[7]}$ attributed the alignment of $\alpha^{\prime}$ to the ratio of the shear modules between the precipitates and

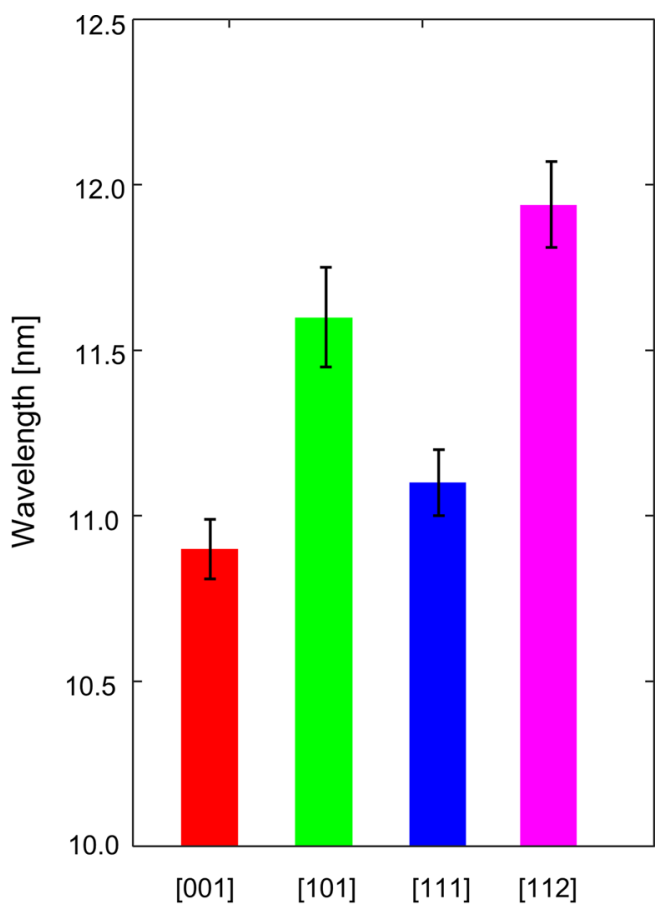

(b)

Fig. 7- Orientation-dependent wavelength in (a) QA and (b) APT when a uniaxial tensile load of 231 and 235 MPa is applied, respectively. 
matrix, given that $\alpha^{\prime}$ precipitates in $\mathrm{Fe}-\mathrm{Cr}$ alloys are soft. A soft precipitate will align itself perpendicular to the applied tensile strain. Li et al. ${ }^{[34]}$ predicted directional growth through effective eigenstrain; a direction with lower eigenstrain is generally a favorable direction for decomposition to proceed. In the case of $\mathrm{Fe}-\mathrm{Cr}$ alloys, $\varepsilon_{x x}<0$ and $\varepsilon_{y y}>0$; thus, eigenstrain is compressive in the $x$-axis and tensile in the $y$-axis. Therefore, elongation occurs in the $y$-axis perpendicular to an applied load in the $z$-direction. This is in line with our observations in QA and APT (Figure 5); the merit of using QA is to maintain true atomic resolution and crystal structure in the analysis of nanostructural segregation on the continuum time scale without assuming concentration-dependent elastic properties.

Treatment of anisotropy of drift diffusion due to elastic strain fields in cubic crystals requires consideration when comparing results from different orientations. ${ }^{[42]}$ In the presence of an external force field, the diffusion current density needs to consider drift current, which is proportional to the gradient of the interaction energy. The symmetry of the host lattice can strongly affect the symmetry of the elementary-jump mechanism of the defect (e.g., vacancy) itself. The addition of a continuous strain field alters the saddle point in the activation energy barrier and jump rates in different directions. In addition, the diffusion constant itself is a hyperbolic strain-dependent coefficient. ${ }^{[43]}$ The migration energy of diffusion in a force field is proportional to the gradient energy between the equilibrium energy and the saddle point; the external field affects the saddle point and the equilibrium energy remains unchanged. Under normal conditions, the external strain is small so that it can be linearly approximated. Thus, the expected effect of uniaxial loading in different crystallographic directions on the diffusion constant is only a few percent.

1. The effect of applied strain can be modeled at the atomic scale using the QA approach. For the first time, the real strain in the system was quantified and directly compared with experimental data. Thus, different strain tensors and rotation matrices are introduced to alter the stress states. This approach has shown promising results for tensile, compression, and shear stress in addition to the rotation of the crystal structure. Introducing an infinitesimal displacement of an atom triggers a free energy response of the system to alter its morphology in good agreement with APT. In the case of Fe-Cr, high-load levels are required to have a noticeable morphological anisotropy effect near the elastic limit.

2. The added external load does affect the diffusion-controlled decomposition process in the experiment. The addition of elastic load in the Fe-35 at. pct $\mathrm{Cr}$ system only has minor effects dependent on the differences in the crystallographic orientation. Still, the close-packed [101] orientation favors decomposition in these experiments, indicating that the ease of diffusion is more important than the difference in elastic properties in the direction of the applied load between the different orientations.
3. The exact stress state in the polycrystalline materials is still unknown. However, it is unlikely to have a dramatic effect on these results. It should be mentioned that there is still room for improvement in the treatment of the polycrystalline materials.

\section{ACKNOWLEDGMENTS}

The financial support from the Centre National de la Recherche Scientifique (CNRS), Region-Normandie, EIT Raw Materials project ENDUREIT, and Carl Tryggers Research Foundation is gratefully acknowledged. This work was partly carried out owing to the experimental GENESIS platform. GENESIS is supported by the region Haute Normandie, the Metropole Rouen Normandie, the CNRS via LABEX EMC, and the French National Research Agency as part of the program "Investissement d'avenir" ARN-11-EQPX-0020. The authors thank Drs. A Vaugeois, C. Keller, and A. Guillet for their support and discussion of this work.

\section{CONFLICT OF INTEREST}

The authors declare that they have no known competing financial interests or personal relationships that could have appeared to influence the work reported in this article.

\section{DATA AVAILABILITY}

All datasets, code, and results occupy several gigabytes of data, which are available from the authors upon serious request.

\section{FUNDING}

Open access funding provided by Royal Institute of Technology.

\section{OPEN ACCESS}

This article is licensed under a Creative Commons Attribution 4.0 International License, which permits use, sharing, adaptation, distribution and reproduction in any medium or format, as long as you give appropriate credit to the original author(s) and the source, provide a link to the Creative Commons licence, and indicate if changes were made. The images or other third party material in this article are included in the article's Creative Commons licence, unless indicated otherwise in a credit line to the material. If material is not included in the article's Creative Commons licence and your intended use is not permitted by statutory regulation or exceeds the permitted use, you will need to obtain permission directly from the copyright holder. To view a copy of this licence, visit http://creat ivecommons.org/licenses/by/4.0/. 


\section{APPENDIX}

Calculated stereographic projections of the principal directions of bcc Fe.

$\left(\begin{array}{lll}0 & 0 & 1\end{array}\right) /\left[\begin{array}{lll}0 & 0 & 1\end{array}\right]$

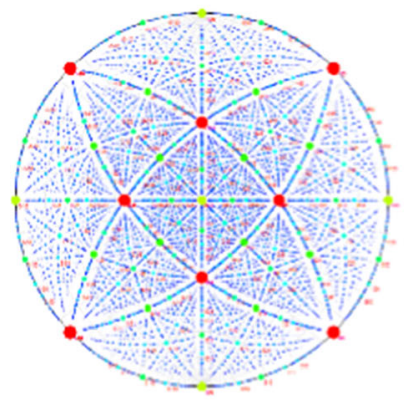

$\left(\begin{array}{lll}1 & 0 & 1\end{array}\right) /\left[\begin{array}{lll}1 & 0 & 1\end{array}\right]$

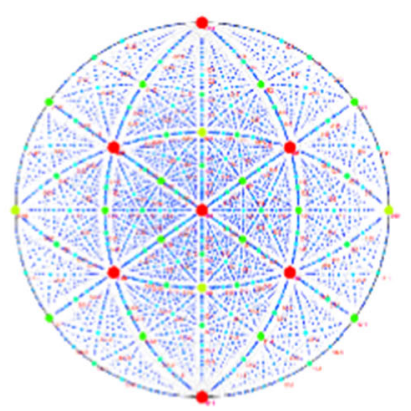

$\left(\begin{array}{lll}1 & 1 & 1\end{array}\right) /\left[\begin{array}{lll}1 & 1 & 1\end{array}\right]$

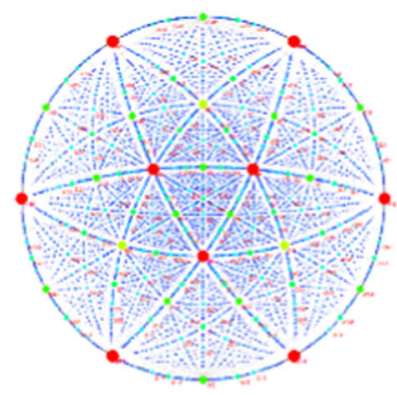

$\left(\begin{array}{lll}1 & 1 & 2\end{array}\right) /\left[\begin{array}{lll}1 & 1 & 2\end{array}\right]$

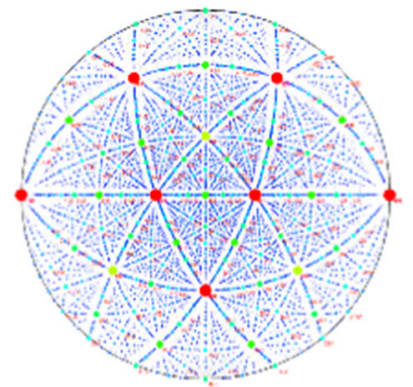

The bcc Fe stereographic projections were calculated using the WinWulff 1.6, Stereographic Projection Software, @JCrystalSoft, 2006-2018.

\section{REFERENCES}

1. K.R. Elder and M. Grant: Phys. Rev. E, 2004, vol. 70, p. 051605.

2. N. Provatas, P.J.A. Dantzig, B. Athreya, P. Chan, P. Stefanovic, N. Goldenfeld, and KR. Elder: JOM, 2007, vol. 59, pp. 83-90.

3. A.G. Khachaturyan: Theory of Structural Transformations in Solids, Wiley, New York, 1993.

4. M. Lavrskyi, H. Zapolsky, and A.G. Khachaturyan: arXiv:1411. 5587[cond-mat].

5. A.K. da Silva, D. Ponge, Z. Peng, G. Inden, Y. Lu, A. Breen, B. Gault, and D. Raabe: Nat. Commun., 2018, vol. 9, p. 1137.

6. G. Radnóczi, E. Bokányi, Z. Erdélyi, and F. Misják: Acta Mater., 2017, vol. 123, pp. 82-89.

7. L. Zhu, Y. Li, C. Liu, S. Chen, S. Shi, and S. Jin: Model. Simul. Mater. Sci. Eng., 2018, vol. 26, p. 035015.

8. X. Xu, J. Odqvist, MH. Colliander, S. King, M. Thuvander, A. Steuwer, and P. Hedström: Acta Mater., 2017, vol. 134, pp. 221-29.

9. S.M. Wise, J.S. Kim, and W.C. Johnson: Thin Solid Films, 2005, vol. 473, pp. 151-63.

10. A. Hörling, L. Hultman, M. Odén, J. Sjölén, and L. Karlsson: Surf. Coat. Technol., 2005, vol. 191, pp. 384-92.

11. F. Danoix and P. Auger: Mater. Charact., 2000, vol. 44, pp. 177-201.

12. N. Pettersson, S. Wessman, M. Thuvander, P. Hedström, J. Odqvist, RFA. Pettersson, and S. Hertzman: Mater. Sci. Eng. A, 2015, vol. 647, pp. 241-48.

13. J. Zhou, J. Odqvist, M. Thuvander, S. Hertzman, and P. Hedström: Acta Mater., 2012, vol. 60, pp. 5818-27.

14. J.W. Cahn: General Electric Research Laboratory, Schenectady, NY.

15. M.E. Thompson and P.W. Voorhees: Model. Simul. Mater. Sci. Eng., 1997, vol. 5, pp. 223-43.

16. C. Pareige, J. Emo, S. Saillet, C. Domain, and P. Pareige: J. Nucl. Mater., 2015, vol. 465, pp. 383-89.

17. C. Pareige, S. Novy, S. Saillet, and P. Pareige: J. Nucl. Mater., 2011, vol. 411, pp. 90-96.

18. W. Xiong, K.A. Grönhagen, J. Ågren, M. Selleby, J. Odqvist, and Q. Chen: Solid State Phenom., vols. 172-174, pp. 1060-65.

19. F. Danoix, J. Lacaze, A. Gibert, D. Mangelinck, K. Hoummada, and E. Andrieu: Ultramicroscopy, 2013, vol. 132, pp. 193-98.

20. L.-Q. Chen and A.G. Khachaturyan: Acta Metall. Mater., 1991, vol. 39 , pp. $2533-51$

21. G. Demange, M. Lavrskyi, K. Chen, X. Chen, Z.D. Wang, R. Patte, and H. Zapolsky: arXiv:2103.12384[cond-mat].
22. N. Mavrikakis, C. Detlefs, P.K. Cook, M. Kutsal, A.P.C. Campos, M. Gauvin, P.R. Calvillo, W. Saikaly, R. Hubert, H.F. Poulsen, A. Vaugeois, H. Zapolsky, D. Mangelinck, M. Dumont, and C. Yildirim: Acta Mater., 2019, vol. 174, pp. 92-104.

23. H. Zapolsky, A. Vaugeois, R. Patte, and G. Demange: Materials, 2021, vol. 14, p. 4197.

24. O. Kapikranian, H. Zapolsky, R. Patte, C. Pareige, B. Radiguet, and P. Pareige: Phys. Rev. B.

25. M. Lavrskyi, H. Zapolsky, and A.G. Khachaturyan: NPJ Comput. Mater., 2016, vol. 2, pp. 1-9.

26. H. Zapolsky: Order, Disorder and Criticality, World Scientific, Singapore, 2014, pp. 153-92.

27. M. Lavrskyi: Thesis, Normandie, 2017.

28. M. Müller, P. Erhart, and K. Albe: J. Phys.: Condens. Matter, 2007, vol. 19, p. 326220.

29. M.I. Mendelev, S. Han, D.J. Srolovitz, G.J. Ackland, D.Y. Sun, and M. Asta: Philos. Mag., 2003, vol. 83, pp. 3977-94.

30. R. Pasianot, D. Farkas, and E.J. Savino: Phys. Rev. B, 1991, vol. 43, pp. 6952-61.

31. A. Gueddouh, B. Bentria, Y. Bourourou, and S. Maabed: Mater. Sci.-Poland, 2016, vol. 34, pp. 503-16.

32. G. Huang, Q. Zhang, and S. Li: Mater. Res. Express, 2020, vol. 7, p. 076509 .

33. M.P. Moody, B. Gault, L.T. Stephenson, D. Haley, and S.P. Ringer: Ultramicroscopy, 2009, vol. 109, pp. 815-24.

34. Y. Li, Y. Yu, X. Cheng, and G. Chen: Mater. Sci. Eng. A, 2011, vol. 528, pp. 8628-34.

35. Y.-T. Chiu, C.-K. Lin, and J.-C. Wu: J. Power Sources, 2011, vol. 196, pp. 2005-12.

36. G.I. Taylor: J. Inst. Met., 1938, vol. 62, pp. 307-24.

37. J.W. Cahn: J. Chem. Phys., 1965, vol. 42, pp. 93-99.

38. A. Vidyasagar, S. Krödel, and D.M. Kochmann: Proc. R. Soc. A, 2018, vol. 474, p. 20180535.

39. J.W. Cahn: Acta Metall., 1961, vol. 9, pp. 795-801.

40. S.M. Dubiel and G. Inden: Z. Metallkd., 1987, vol. 78, p. 544.

41. J. Zhou, J. Odqvist, M. Thuvander, and P. Hedström: Microsc. Microanal., 2013, vol. 19, pp. 665-75.

42. P.H. Dederichs and K. Schroeder: Phys. Rev. B, 1978, vol. 17, pp. 2524-36.

43. A.V. Nazarov and A.A. Mikheev: J. Phys.: Condens. Matter, 2008, vol. 20, p. 485203.

Publisher's Note Springer Nature remains neutral with regard to jurisdictional claims in published maps and institutional affiliations. 